\title{
Autonomic Nerve Regulation of Colonic Peristalsis in Guinea Pigs
}

\author{
Irena Gribovskaja-Rupp, Reji Babygirija, Toku Takahashi* and Kirk Ludwig \\ Department of Surgery, Medical College of Wisconsin and Zablocki VA Medical Center, Milwaukee, WI, USA
}

\begin{abstract}
Background/Aims
Colonic peristalsis is mainly regulated via intrinsic neurons in guinea pigs. However, autonomic regulation of colonic motility is poorly understood. We explored a guinea pig model for the study of extrinsic nerve effects on the distal colon.
\end{abstract}

\section{Methods}

Guinea pigs were sacrificed, their distal colons isolated, preserving pelvic nerves (PN) and inferior mesenteric ganglia (IMG), and placed in a tissue bath. Fecal pellet propagation was conducted during PN and IMG stimulation at $10 \mathrm{~Hz}, 0.5 \mathrm{~ms}$ and $5 \mathrm{~V}$. Distal colon was connected to a closed circuit system, and colonic motor responses were measured during PN and IMG stimulation.

\section{Results}

PN stimulation increased pellet velocity to $24.6 \pm 0.7 \mathrm{~mm} / \mathrm{sec}(n=20)$, while IMG stimulation decreased it to $2.0 \pm 0.2$ $\mathrm{mm} / \mathrm{sec}(\mathrm{n}=12)$, compared to controls $(13.0 \pm 0.7 \mathrm{~mm} / \mathrm{sec}, P<0.01)$. In closed circuit experiments, PN stimulation increased the intraluminal pressure, which was abolished by atropine $\left(10^{-6} \mathrm{M}\right)$ and hexamethonium $\left(10^{-4} \mathrm{M}\right)$. PN stimulation reduced the incidence of non-coordinated contractions induced by NG-nitro-L-arginine methyl ester (L-NAME; 10 $\left.{ }^{-4} \mathrm{M}\right)$. IMG stimulation attenuated intraluminal pressure increase, which was partially reversed by alpha-2 adrenoceptor antagonist (yohimbine; $10^{-6} \mathrm{M}$ ).

\section{Conclusions}

PN and IMG input determine speed of pellet progression and peristaltic reflex of the guinea pig distal colon. The stimulatory effects of PN involve nicotinic, muscarinic and nitrergic pathways. The inhibitory effects of IMG stimulation involve alpha-2 adrenoceptors.

\section{(J Neurogastroenterol Motil 2014;20:185-196)}

\section{Key Words}

Colonic motility; Pelvic nerve; Inferior mesenteric ganglia; Nitric oxide

Received: October 18, 2013 Revised: December 28, 2013 Accepted: December 29, 2013

(c) This is an Open Access article distributed under the terms of the Creative Commons Attribution Non-Commercial License (http://creativecommons. org/licenses/by-nc/3.0) which permits unrestricted non-commercial use, distribution, and reproduction in any medium, provided the original work is properly cited.

*Correspondence: Toku Takahashi, MD, PhD

Financial support: None.

Department of Surgery, Zablocki VA Medical Center, 5000 West National Avenue, Milwaukee, WI 53295, USA

Tel: +1-414-384-2000 (ext. 41472), Fax: +1-414-382-5374, E-mail: ttakahashi@mcw.edu

Conflicts of interest: None.

Author contributions: Toku Takahashi and Irena Gribovskaja-Rupp designed the research; Irena Gribovskaja-Rupp and Reji Babygirija performed the experiments and analyzed data; Irena Gribovskaja-Rupp, Reji Babygirija and Toku Takahash wrote the manuscript; Toku Takahash and Kirk Ludwig contributed to critical revision. 


\section{Introduction}

Damage to the autonomic efferent nerves in the pelvis results in colonic dysmotility states. Radical hysterectomy causes significant disturbance of urinary and anorectal function, and $42 \%$ of patients report significant post-operative bowel dysfunction. ${ }^{1}$ The etiology is extrinsic autonomic denervation, as demonstrated by loss of anorectal inhibitory reflex, regulated by the autonomic system, in $64 \%$ of patients. ${ }^{2}$ Low anterior resection syndrome, exhibited by $90 \%$ of patients after major rectal resection, also has features consistent with autonomic damage. Internal anal sphincter dysfunction is consistently found in low anterior resection syndrome, and does not recover over time, likely due to autonomic nerve damage during rectal mobilization. ${ }^{3}$ Neurogenic fecal incontinence is another disorder generated by abnormal autonomic innervation. ${ }^{4}$

Sacral nerve stimulation, a prominent therapy with 75-100\% sustained success rate for fecal incontinence, ${ }^{5}$ is based on extrinsic direct manipulation of the autonomic nervous system at the sacral root. Interestingly, the same therapy is also used to treat constipation, with comparable success rates. ${ }^{6}$ Its mechanisms of action are poorly understood and include normalization of colonic transit time. ${ }^{6}$

Design of cost-effective animal models that can be easily manipulated has been a challenge. Denervation of rectum in rats has been shown to produce abnormal hyper-motility in the neorectum in the early post-operative phase, which was associated with increased acetylcholine $(\mathrm{ACh})$ and neuronal nitric oxide synthase (NOS) content in the myenteric plexus. ${ }^{7}$ Another study also showed that surgical denervation of the left colon in rats resulted in a significant increase in motility. ${ }^{8}$ Transection of rectal branches of the pelvic plexus in dogs resulted in partial recovery of transit time and defecation reflex months after denervation, implying that a compensatory mechanism was involved. ${ }^{9}$ Our studies of compensatory mechanisms that are at play after autonomic nerve transection in a rat model have shown that transection of both sympathetic and parasympathetic nerve supply to the colon acutely affected motility, but that recovery occurred at a week post-operatively. ${ }^{10}$ Mechanisms responsible for recovery of delayed colonic motility involved upregulation of 5-hydroxytryptamine $3\left(5-\mathrm{HT}_{3}\right)^{11}$ and $5-\mathrm{HT}_{4}$ receptors. ${ }^{12}$

Parasympathetic innervation of the guinea pig distal colon comes from sacral S2-S4 roots as pelvic nerves (PN) and coalesces in anterior and posterior pelvic ganglia to distribute to the rectum as rectal nerves. ${ }^{13}$ The anterior pelvic ganglia are located bilaterally adherent to the lateral aspect of the proximal part of the seminal vesicles, in the angle between ductus deference and the seminal vesicle. ${ }^{14}$ The posterior ganglion contributes to a much smaller extent to efferent innervation compared to the anterior ganglion. ${ }^{13,15}$ The anterior pelvic ganglia send and receive projections from the inferior mesenteric ganglion (IMG) via hypogastric nerves, thus making it a mixed parasympathetic/sympathetic structure, as it is in the rat. ${ }^{15}$ The majority of anterior ganglion projections travel to the rectum and not the distal colon. ${ }^{13}$ The majority of parasympathetic supply to the colon comes from the hypogastric nerves and transection of pelvic and hypogastric nerves is necessary to eliminate extrinsic parasympathetic supply to the colon and rectum. ${ }^{16}$ Conversely, sympathetic supply to the distal colon comes from the L2-L4 lumbar spinal cord as splanchnic nerves, coalesces in the prevertebral ganglia (such as celiac, superior mesenteric and IMG) then distributes as colonic nerves that closely follow the vascular supply. ${ }^{13}$ The IMG forms a ring around inferior mesenteric artery (IMA) shortly after IMA takeoff from the aorta, and an intermesenteric nerve that connects IMG to superior mesenteric ganglia, as well as hypogastric nerves that connect IMG to anterior pelvic ganglion, coalesce on this ring (intraoperative observation).

Denervation studies of the guinea pig colon have been performed to date to examine effect on opioid receptors by way of performing short cryoablation of IMA/IMG complex, the so called "frigore" denervation of the IMG, which destroys the IMG but preserves IMA, ${ }^{17-19}$ and histological and immunohistochemical changes in the pelvic ganglia after denervation. ${ }^{16,20}$

To create a model for the study of isolated extrinsic nerve stimulation on the distal colon, we chose guinea pigs due to the anatomic complexity of pelvic innervation and active enteric nervous system, which is well established for the study of intrinsic reflexes. We have designed an in vitro model of guinea pig distal colon that allows full manipulation of the PN and IMG in vitro. Our goals were 3-fold: first, design an effective model to study the effects of extrinsic nerve manipulation on distal colon; second, quantify the particular effects of extrinsic nerve stimulation on the behavior of the distal colon; third, describe the possible mechanisms of extrinsic nerve stimulation and its effects.

\section{Materials and Methods}

\section{Animals}

Male Hartley guinea pigs (body weight 300-350 g) were ob- 
tained from Charles River Laboratories (Durham, NC, USA). The animals were housed in a controlled environment $\left(21 \pm 1^{\circ} \mathrm{C}\right.$, $50-70 \%$ humidity, 12-hour light dark cycle) and given free access to food and water. They were housed at standard conditions for at least 7 days prior to any experimentation.

Animals were maintained and handled in accordance with policies of the Animal Care and Use Committee of Clement J. Zablocki VA Medical Center (Milwaukee, WI, USA). All the experimental protocols were approved by same institution and in accordance with the National Institutes of Health Guide for the Care and Use of Laboratory Animals.

\section{Dissection of the Pelvic Parasympathetic and Sympathetic Nerves}

Animals were anesthetized by isoflurane (2\%). Midline laparotomy was performed and the small intestine was eviscerated. Colon was gently rotated laterally from the abdominal cavity. Retractors were applied to pelvic side walls. Sacral nerves coalesced into anterior pelvic ganglion on the posterior portion of the prostate bilaterally, as described, ${ }^{13-15}$ and were transected at the point of entry into the ganglion. Posterior pelvic ganglion was not dissected because it represented $10 \%$ of efferent neuron connections. ${ }^{15}$ Next, IMA was identified, and the IMG forming a ring around it was visualized IMA/IMG complex was carefully dissected and ligated just distal to IMA take-off from the abdominal aorta. Finally, colon was transected $1-2 \mathrm{~cm}$ proximal to IMA/IMG complex and distally just beyond the prostate/bladder complex, preserving the mesentery. Following dissection the animals were euthanized with isoflurane (5\%). Care was taken to accomplish the dissection in 10 minutes and avoid any intraluminal blood spillage. The dissection of $\mathrm{PN}$ and IMG was performed under the dissecting microscope and 4-0 silk suture was used to tag all nerves for ease of identification in the tissue bath. All pellets contained in the colonic segment were cleared by a brief $\mathrm{PN}$ stimulation upon placement of colons into the tissue bath.

\section{Fecal Pellet Propulsion Study In Vitro}

The distal colon was excised from the lower pole of the left kidney to the pelvic brim. Colonic segments (6 $\mathrm{cm}$ in length) were laid horizontally in the organ bath filled with Krebs-Henseleit buffer (KHB) $\left(\mathrm{MgSO}_{4} 1.2 \mathrm{mM}, \mathrm{KH}_{2} \mathrm{PO}_{4} 1.2 \mathrm{mM}, \mathrm{KCl}\right.$ $4.8 \mathrm{mM}, \mathrm{NaCl} 118 \mathrm{mM}, \mathrm{NaHCO}_{3} 25 \mathrm{mM}, \mathrm{CaCl}_{2} 2.5 \mathrm{mM}$ and D-glucose $11 \mathrm{mM}$ ) at $37^{\circ} \mathrm{C}$ and bubbled with $95 \% \mathrm{O}_{2}$ and $5 \%$ $\mathrm{CO}_{2}$. The colonic segments were fixed to the bottom of the bath with an acupuncture pin at the oral and anal ends so as not to obstruct or distend the lumen. After a 30-minute equilibration, a natural fecal pellet ( $4 \mathrm{~mm}$ in diameter and $10 \mathrm{~mm}$ in length) coated with nail polish was inserted into the oral end. The pellet propulsion was measured by monitoring the time it took for the pellet to traverse the colonic segments via a video recording. The studies were repeated 5-10 times at 5-minute intervals. In order to appreciate the effect of nerve stimulation, a nerve stimulation probe (AD Instruments, Colorado Springs, CO, USA) was attached to either the IMG or one of the PN just beyond the suture. To determine whether a lower or higher frequency can elicit the optimal response to $\mathrm{PN}$ stimulation, we applied electrical stimulation at various frequencies $(1,2,5,10$ and $20 \mathrm{~Hz}, 0.5 \mathrm{~ms}$ and $5 \mathrm{~V}$ ) for 10 minutes maximum or until pellet was expelled by the colonic tissue (usually 10-50 seconds).

\section{Intraluminal Pressure Recording In Vitro}

As previously reported, colonic segments (6 $\mathrm{cm}$ in length) were laid horizontally in the organ bath filled with $\mathrm{KHB} .{ }^{11,21,22}$ The oral end of the segment was connected to an infusion syringe, and the anal end was connected to a pressure transducer (AD Instruments). After equilibration of 30 minutes, $0.3 \mathrm{~mL}$ $\mathrm{KHB}$ was slowly infused for 30 seconds through the oral end. The colonic segments were kept distended with KHB for 10 minutes. Then, luminal content was removed and tissues were allowed 20 minutes for equilibration without distension. Similar distension studies were repeated 5-7 times. Our recent study showed that $0.3 \mathrm{~mL}$ of luminal infusion was able to initiate motor responses with concomitant increase of intraluminal pressure in guinea pigs. Motor responses and intraluminal pressure changes were consistent at least 5-7 times in each tissue in response to luminal infusion. ${ }^{22}$ Intraluminal pressure changes in response to luminal infusion were recorded by a software system (Power-Lab model 8SP; AD Instruments). Motor responses to luminal infusion were recorded. If peristaltic waves propagated across at least half of the colonic segment, this motor response was defined as a normal peristaltic contraction (PC). ${ }^{22}$ In response to luminal distension, various types of motor responses were observed, such as localized contractions, or partially propagated contractions. These motor responses were defined as non-peristaltic contractions (NPCs). The incidence of PCs was more than $82 \%$ of the guinea pig distal colon in response to luminal infusion $(0.3$ $\mathrm{mL})^{22}$

The frequency (the number of contractions per minute), the amplitude (difference between the baseline and the peak of the in- 
transluminal pressure), and the motility index (MI, area under the curve expressed as $\mathrm{cmH}_{2} \mathrm{O} \cdot \mathrm{sec}$ ) were analyzed and regional differences were compared. Ten minutes prior to PN stimulation, atropine $\left(10^{-6} \mathrm{M}\right)$, hexamethonium $\left(10^{-4} \mathrm{M}\right)$, or NG-nitro-L-arginine methyl ester (L-NAME; $10^{-4} \mathrm{M}$ ) were added to the tissue bath. Ten minutes prior to IMG stimulation, yohimbine $\left(10^{-6} \mathrm{M}\right)$ was added to the tissue bath.

\section{Chemicals}

L-NAME was purchased from Tocris Bioscience (Bristol, UK). Atropine, hexamethonium, and yohimbine were obtained from Sigma Chemical Company (St. Louis, MN, USA).

\section{Statistical Methods}

Data were shown as means \pm SE. Student's $t$ test was used to compare differences in continuous variables between the two groups. An analysis of variance with post-hoc Duncan test was used to compare the regional differences of the colon. Statistical significance was defined as $P<0.05$. $\mathrm{N}$ refers to number of animals used for each test.

\section{Results}

\section{Fecal Pellet Propulsion Study}

In the distal colon, the fecal pellet was expelled within 2 mi-

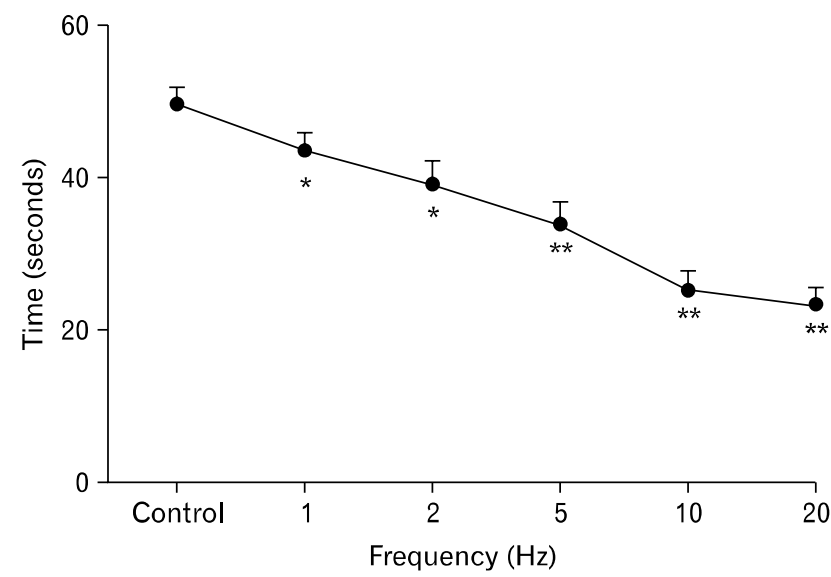

Figure 1. Pellet propulsion time in response to pelvic nerves (PN) stimulation $(1-20 \mathrm{~Hz})$. Pellet propulsion time was significantly shortened in a frequency dependent manner. Submaximal effects were observed at $10 \mathrm{~Hz}(24.5 \pm 2.3$ seconds, $\mathrm{n}=6)$, compared to control group (without nerve stimulation, $49.8 \pm 1.8$ seconds, $\mathrm{n}=6)\left({ }^{*} P<\right.$ $\left.0.05,{ }^{* *} P<0.01\right)$. nutes in all colons that were tested. No retrograde propagation of the pellet was observed. The average velocity of pellet propulsion was $13.0 \pm 0.7 \mathrm{~mm} / \mathrm{sec}(\mathrm{n}=20)$. PN stimulation significantly shortened the pellet propulsion time in a frequency dependent manner (1-20 Hz) (Fig. 1). The velocity of pellet propagation increased significantly in response to $\mathrm{PN}$ stimulation $(10 \mathrm{~Hz})$ to $24.6 \pm 0.7 \mathrm{~mm} / \mathrm{sec}(\mathrm{n}=20, P<0.01)$. In contrast, pellet propulsion was significantly delayed during IMG stimulation (10 $\mathrm{Hz}$ ) to $2.0 \pm 0.2 \mathrm{~mm} / \mathrm{sec}(\mathrm{n}=12)$ (Fig. 2 and Supplementary Videos 1-3). In 8 of 20 colons, no pellet propagation occurred at all and pellet was never expelled.

\section{Intraluminal Pressure Recording}

In the presence of $\mathrm{PN}$ stimulation, frequency and magnitude of intraluminal pressure were increased, and returned to baseline as soon as nerve stimulation ceased (Fig. 3). The opposite phenomenon was observed during IMG stimulation. No pressure increase was recorded during intraluminal fluid infusion during IMG stimulation, and tissue promptly returned to normal response once IMG stimulation ceased (Fig. 3).

We quantified these changes by measuring MI (Fig. 4A), frequency (Fig. 4B), duration (Fig. 4C) and amplitude (Fig. 4D) of intraluminal pressure responses to luminal infusion $(0.3$ $\mathrm{mL}$ ). PN stimulation produced MI (percent of control) that was $151.0 \pm 5.3 \%$ of controls ( $\mathrm{n}=10, P<0.01)$, while IMG stimulation was associated with $\mathrm{MI}$ that was $16.0 \pm 2.3 \%$ of controls

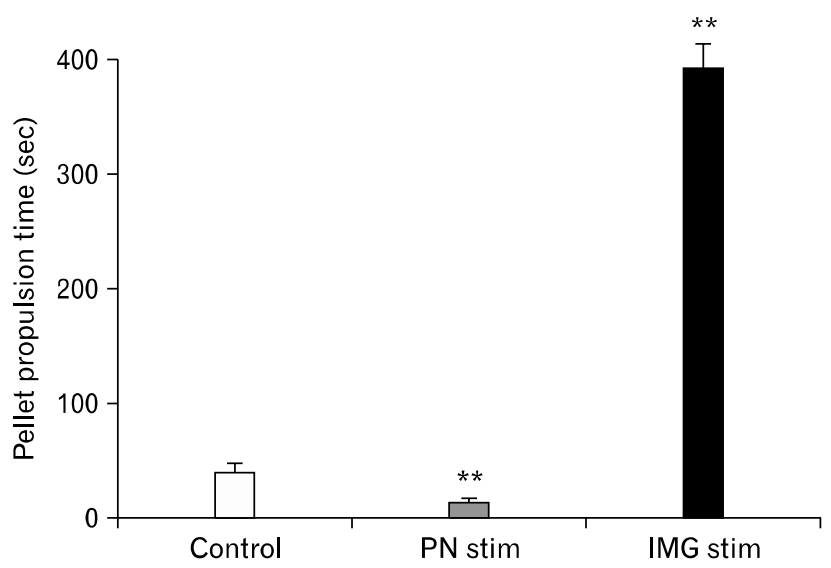

Figure 2. Pellet propagation velocity during autonomic nerve stimulation. Pellet propagation velocity was significantly increased during pelvic nerves $(\mathrm{PN})$ stimulation to $24.6 \pm 0.7 \mathrm{~mm} / \mathrm{sec}(\mathrm{n}=20$, $\left.{ }^{* *} P<0.01\right)$ and decreased during inferior mesenteric ganglia (IMG) stimulation to $2.0 \pm 0.2 \mathrm{~mm} / \mathrm{sec}\left(\mathrm{n}=12,{ }^{* *} \mathrm{P}<0.01\right)$, compared to control group (without nerve stimulation, $13.0 \pm 0.7 \mathrm{~mm} / \mathrm{sec}, \mathrm{n}=20$ ). 
A

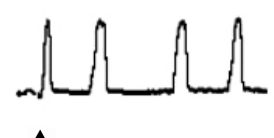

B

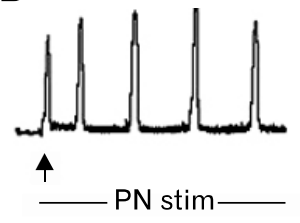

$\left.\iint\right\}$

4

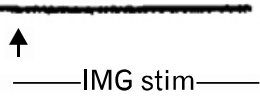

C
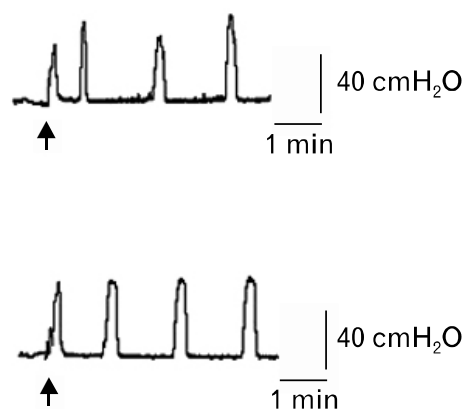

Figure 3. Intraluminal pressure recording before (A), during (B) and after (C) pelvic nerves (PN; upper panel) and inferior mesenteric ganglia (IMG; lower panel) stimulation. In response to luminal infusion of $0.3 \mathrm{~mL}$ (indicated by arrows), intraluminal pressure increase was observed in the distal colon. PN stimulation augmented the intraluminal pressure increase, while IMG stimulation abolished intraluminal pressure increase in response to luminal infusion $(0.3 \mathrm{~mL})$.
A
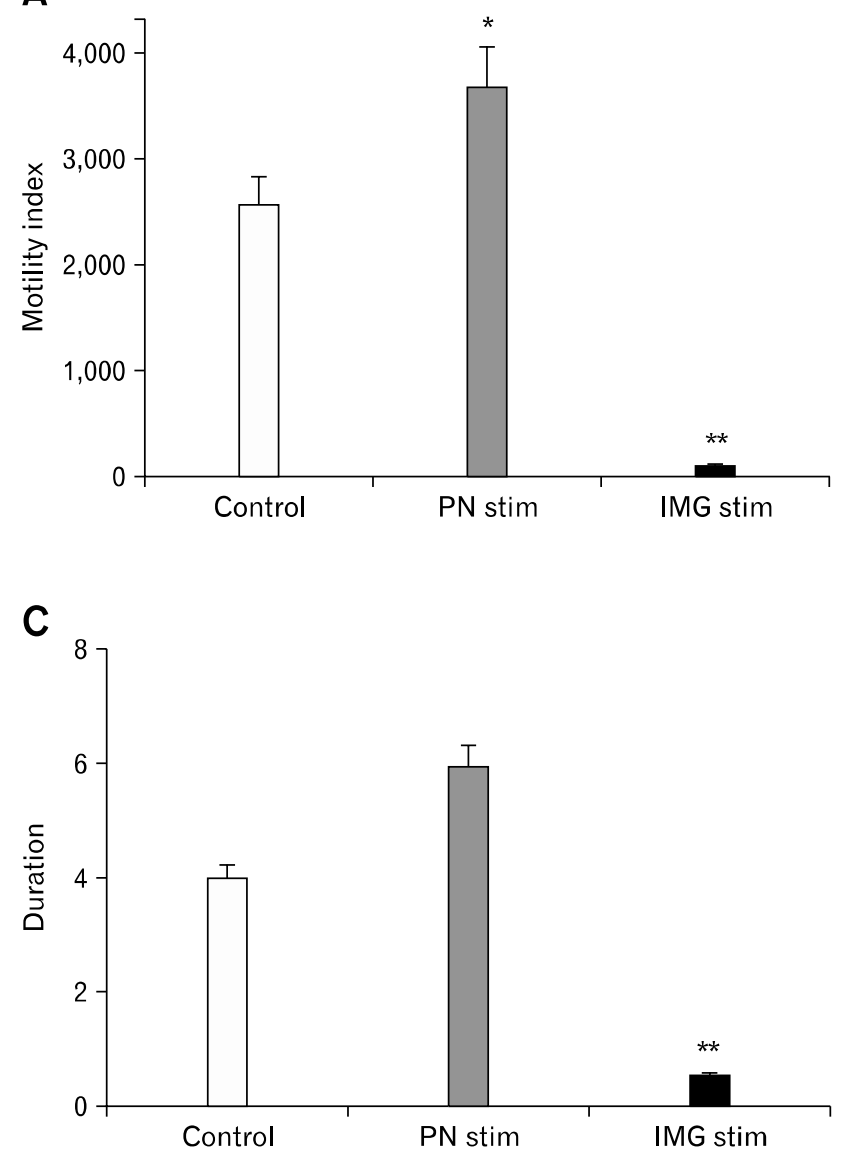

B

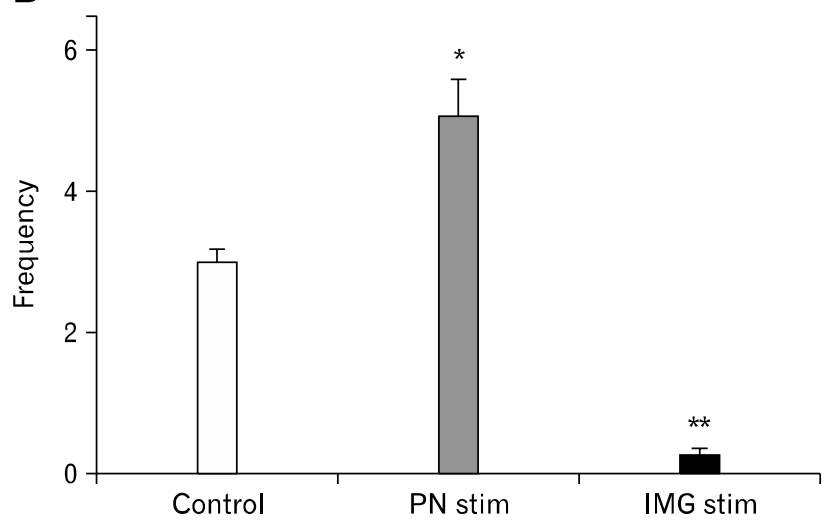

D

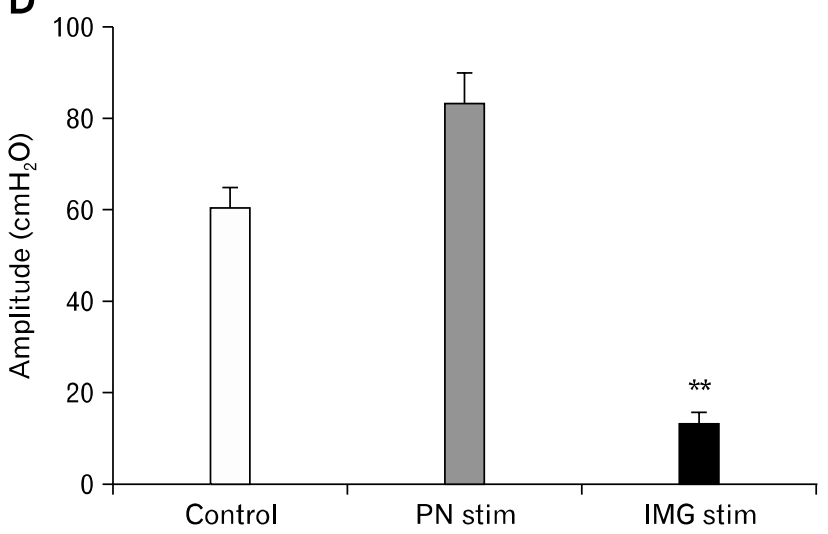

Figure 4. Effects of pelvic nerves (PN) and inferior mesenteric ganglia (IMG) stimulation on motility index (MI) (A), frequency (B), duration (C) and amplitude (D) of intraluminal pressure responses to luminal infusion $(0.3 \mathrm{~mL})$. PN stimulation significantly increased $\mathrm{MI}$ and frequency $(\mathrm{n}=$ $\left.10,{ }^{*} P<0.05\right)$. In contrast, IMG stimulation significantly reduced $\mathrm{MI}$ and frequency $\left(\mathrm{n}=10,{ }^{* *} \mathrm{P}<0.01\right)$.

(n $=10, P<0.01)$ (Fig. 4 A). Similarly, PN stimulation significantly increased frequency to $5.1 \pm 0.5(\mathrm{n}=10, P<0.05)$, while IMG stimulation significantly reduced frequency to $0.3 \pm$ 0.1 (n $=10, P<0.01)$ when compared to controls $(3.0 \pm 0.2)$
(Fig. 4B). PN stimulation did not show any significant difference in duration (Fig. 4C) and amplitude (Fig. 4D). 
Table. The Incidence of Peristaltic Contractions and Non-peristaltic Contractions in the Presence or Absence of L-NAME in Response to Pelvic Nerve stimulation

\begin{tabular}{lcc}
\hline & $\begin{array}{c}\text { The incidence } \\
\text { of PCs }(\%)\end{array}$ & $\begin{array}{c}\text { The incidence } \\
\text { of NPCs }(\%)\end{array}$ \\
\hline Control & $81.6 \pm 6.1$ & $13.6 \pm 2.1$ \\
Control + L-NAME & $16.8 \pm 5.9$ & $78.5 \pm 6.7$ \\
PN stimulation & $90.8 \pm 2.6$ & $8.8 \pm 2.3$ \\
PN stimulation + L-NAME & $48.6 \pm 6.0^{\mathrm{a}}$ & $44.1 \pm 3.6^{\mathrm{a}}$ \\
\hline
\end{tabular}

${ }^{\mathrm{a}} P<0.01$ vs. control + L-NAME $(\mathrm{n}=6)$.

PCs, peristaltic contractions; NPCs, non-peristaltic contractions; L-NAME, NG-nitro-L-arginine methyl ester; $\mathrm{PN}$, pelvic nerves.

Data are presented as means $\pm \mathrm{SE}$.

\section{Effect of L-NAME, Hexamethonium and Atropine on Pelvic Nerves-stimulated Responses}

The incidence of PCs was reduced while the incidence of NPCs was increased in the presence of L-NAME of the guinea pig distal colon in vitro. When PN stimulation was applied to the colon, some NPCs were reversed to PCs in the presence of L-NAME (Table and Fig. 5).

Hexamethonium and atropine abolished intraluminal pressure increase in response to luminal infusion. PN stimulation failed to cause propagating or non-propagating contractions in the presence of hexamethonium (Fig. 6) and atropine (Fig. 7).

\section{Effect of Yohimbine on Inferior Mesenteric Ganglia-stimulated Responses}

IMG stimulation completely abolished intraluminal pressure increase in response to luminal infusion (Fig. 8C). Addition of yohimbine partially reversed contractile activity during IMG stimulation, returning intraluminal pressure tracing close to normal (Fig. 8D) and reconstituting MI to $70 \%$ of control plus yohimbine (Fig. 8E).

\section{Discussion}

Small animals such as rats ${ }^{23,24}$ and guinea pigs ${ }^{25,26}$ have been widely used to study colonic motility. Species difference among humans, rats, and guinea pigs in mediating colonic peristalsis has been demonstrated in various literature.

It has been shown that the inserted pellets are well advanced distally in vitro preparations of the guinea pig distal colon, but not rat distal colon. ${ }^{27-29}$ Our preliminary study also showed that the
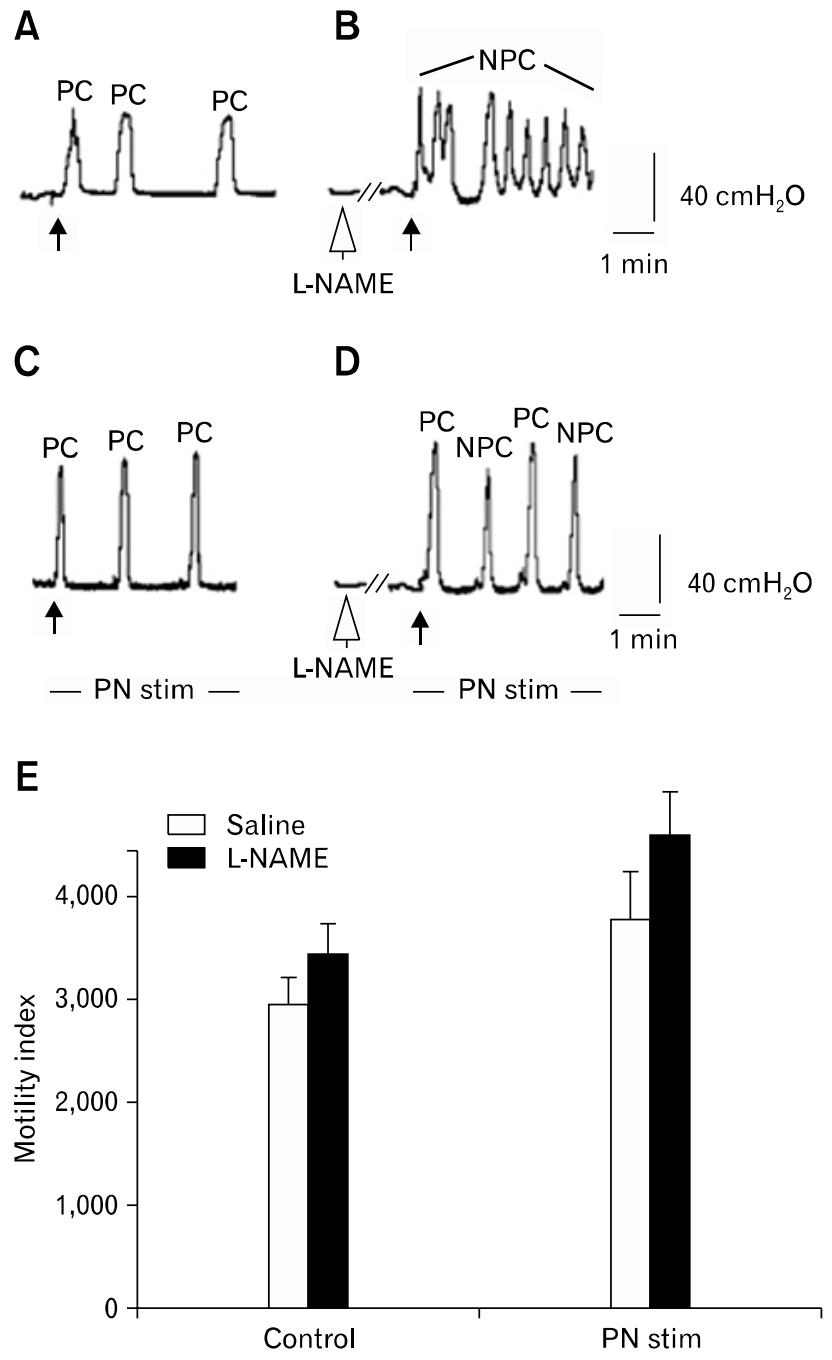

Figure 5. Effects of pelvic nerves $(\mathrm{PN})$ stimulation on intraluminal pressure responses to luminal infusion $(0.3 \mathrm{~mL}$, indicated by arrows) in the absence ( $\mathrm{A}$ and $\mathrm{C}$ ) and presence $(\mathrm{B}$ and $\mathrm{D})$ of NG-nitro-L-arginine methyl ester (L-NAME). Effects of PN stimulation on motility index $(\mathrm{MI})$ in the absence and presence of L-NAME $(\mathrm{E})(\mathrm{n}=6)$. In the presence of L-NAME, the incidence of peristaltic contractions (PCs) was reduced while the incidence of non-peristaltic contractions (NPCs) was increased. When PN stimulation was applied, PCs were reversed to NPC even in the presence of L-NAME. L-NAME itself had no significant effects on MI.

rat distal colon failed to propel fecal pellets in vitro (unpublished observations). Our laboratory has produced a number of studies in a rat model, demonstrating that transection of parasympathetic PN significantly delayed colonic transit time, while the transection of sympathetic hypogastric nerve significantly increased colonic motility. ${ }^{30}$ Response to parasympathetic nerve stimulation was abolished by muscarinic receptor blockade, while response to 
A

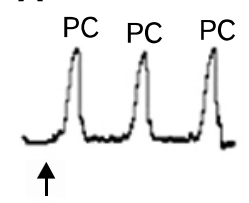

C

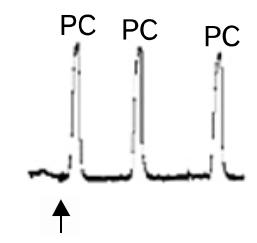

- PN stim -
B

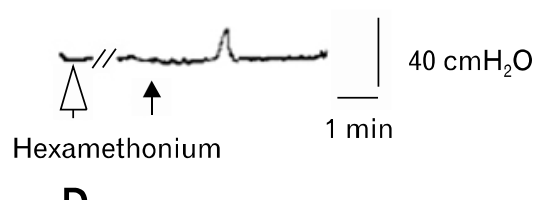

D

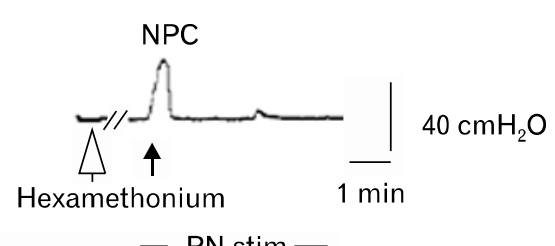

E

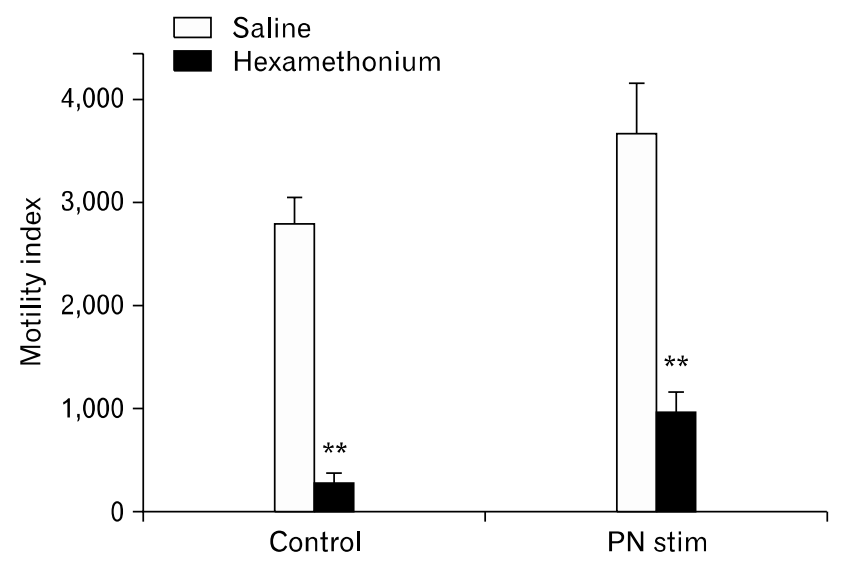

Figure 6. Effects of pelvic nerves (PN) stimulation on intraluminal pressure responses to luminal infusion $(0.3 \mathrm{~mL}$, indicated by arrows $)$ in the absence (A and C) and presence (B and D) of hexamethonium. Effects of $\mathrm{PN}$ stimulation on motility index in the absence and presence of hexamethonium (E) ( $\mathrm{n}=6,{ }^{* *} P<0.01$ compared to saline). Hexamethonium abolished intraluminal pressure increase in response to luminal infusion. PN stimulation failed to cause any peristaltic contractions (PCs) or non-peristaltic contractions (NPCs) in the presence of hexamethonium.

sympathetic nerve stimulation was abolished by beta-adrenoreceptor blockade. ${ }^{31}$ We also showed that the PN dissection significantly impaired colonic transit and colonic motility in rats. ${ }^{10,30}$ Thus, it is likely that extrinsic innervation, rather than intrinsic innervation, plays a major role in regulating colonic peristalsis of the rat colon.

Peristaltic reflexes in response to stretch and mucosal stim-
A

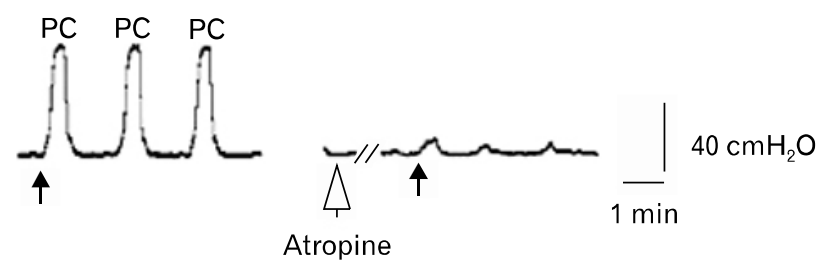

C

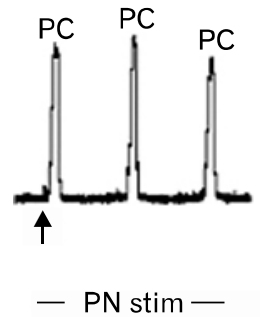

D

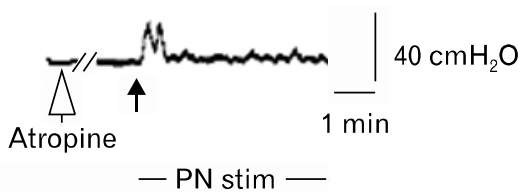

E

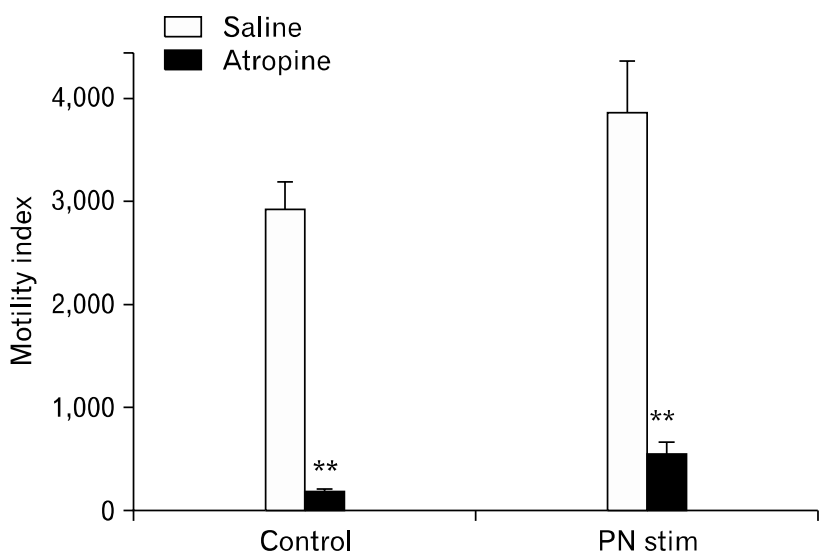

Figure 7. Colonic intraluminal pressure response to luminal infusion $(0.3 \mathrm{~mL}$, indicated by arrows) in the absence $(\mathrm{A}$ and $\mathrm{B})$ and presence $(\mathrm{C}$ and $\mathrm{D}$ ) of pelvic nerves (PN) stimulation, and in the absence (A and C) and presence (B and $\mathrm{D})$ of atropine. Effects of $\mathrm{PN}$ stimulation on motility index in the absence and presence of atropine $(\mathrm{E})\left(\mathrm{n}=6,{ }^{* *} P\right.$ $<0.01$ compared to saline). Atropine abolished intraluminal pressure increase in response to luminal infusion in control colons in the absence of PN stimulation. PN stimulation failed to cause any peristaltic contractions (PCs) or non-peristaltic contractions (NPCs) in the presence of atropine.

ulation are mediated via both extrinsic and intrinsic neurons of the rat colon. ${ }^{32}$ In contrast, both stretch and mucosal reflexes are mediated via intrinsic neurons of the guinea pig colon. ${ }^{33} \mathrm{It}$ is conceivable, therefore, that pellet-stimulated motor responses are less observed in vitro preparations once extrinsic innervation is disrupted in the rat colon. The intrinsic neural pathway mediating peristaltic reflex may be well developed in the guinea pig distal 
A

B

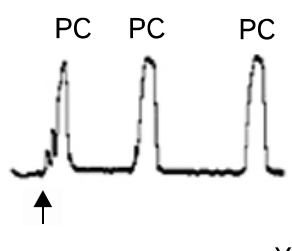

Yohimbine

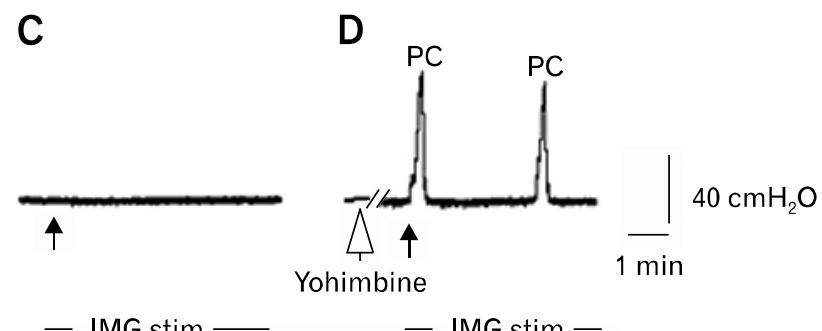

E

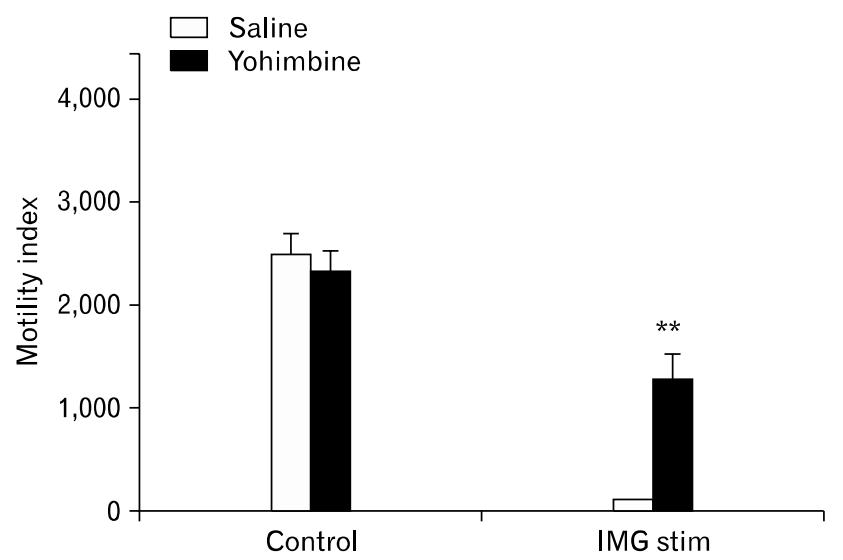

Figure 8. Colonic intraluminal pressure response to luminal infusion $(0.3 \mathrm{~mL}$, indicated by arrows) in the absence ( $\mathrm{A}$ and $\mathrm{B})$ and presence $(\mathrm{C}$ and $\mathrm{D}$ ) of inferior mesenteric ganglia (IMG) stimulation, and in the absence ( $A$ and $C$ ) and presence $(B$ and $D)$ of yohimbine. Effects of IMG stimulation on motility index in the absence and presence of yohimbine $(\mathrm{E})\left(\mathrm{n}=6,{ }^{* *} \mathrm{P}<0.01\right.$ compared to saline). Control colons (A) demonstrate no significant increase in motility index or change in type of contractile activity in response to yohimbine (B). Addition of yohimbine caused return of contractile activity in the presence of IMG stimulation.

colon, compared to rat colon. It is widely accepted that guinea pig peristaltic reflexes are primarily mediated by intrinsic reflexes. ${ }^{33}$ However, it remains unclear how extrinsic neurons regulate peristalsis of the guinea pig colon.

In dogs, prior studies on extrinsic nerve regulation of colonic peristalsis have reported that colonic motility and colorectal reflexes are enhanced by parasympathetic stimulation and inhibited by sympathetic stimulation. ${ }^{34,35}$ The pellet propulsion study demonstrated the overall effect of extrinsic nerve modification of the intrinsic peristaltic reflexes of the guinea pig distal colon. It is the extrinsic nerve input that defines the actual speed of pellet progression through the colon, although it is undeniable that it is the very active enteric nervous sytem of the guinea pig that allows for active pellet propagation even in the absence of extrinsic nerve input.

Our results in guinea pig are similar to in vivo results in the rat, where PN stimulation increased baseline distal colonic motility at least 2-fold and hypogastric nerve stimulation reduced baseline activity, ${ }^{31}$ and transection of these nerves resulted in slowed transit in case of PN and accelerated transit after hypogastric nerve damage. ${ }^{30}$ Our results also correspond to findings in dogs, where damage to PN branches slowed down colonic transit, inhibited defecation reflex, ${ }^{9}$ and resulted in diminished electrical activity. ${ }^{35}$ To our knowledge, this is the first demonstration in guinea pigs that actual changes are observed in colonic pellet propagation velocity in response to PN or IMG stimulation. Extrinsic nerve stimulation affected colonic motility dramatically.

It has been shown that $\mathrm{PN}$ stimulation enhances colonic peristalsis in response to balloon distension while sympathetic stimulation inhibits colonic peristalsis of the guinea pig distal colon in vitro. ${ }^{36,37}$ However, it remains unclear whether PN stimulation affects fecal pellet propulsion or luminal pressure changes. As shown in Supplementary Video 2, we have clearly demonstrated that PN stimulation accelerates pellet propulsion. We also showed that the stimulatory effect of PN stimulation involved muscarinic, nicotinic, and nitrergic pathways. In addition, we also demonstrated that the inhibitory effect of IMG stimulation on colonic peristalsis is mediated, at least in part, via alpha 2 adrenergic receptors.

While there is evidence from animal models ${ }^{7,38}$ and human patients ${ }^{1,39}$ that pelvic and sacral nerve input expedites colonic transit, there are few studies that discuss actual mechanisms of PN stimulation effects on colonic peristalsis. The effects are the following in animal models: internal anal sphincter response is reported as contractile primarily or biphasic consisting of initial relaxation followed by a contraction, whereas colonic response in vivo and in vitro in rabbits and cats produced increased smooth muscle tension ${ }^{40}$ and in dogs produced reduced colonic contraction amplitude. ${ }^{41}$

The high propulsion velocity of a natural pellet might be influenced by the anatomical advantage. In our recent study, the distal colon displayed active and potent motor patterns that are in- 
volved in physiological emptying of the fecal contents. ${ }^{22}$ The recording of pressure changes in response to fluid distension is not identical with normal physiological condition because it keeps the colonic segment evenly distended due to a closed circuit for a certain period (10 minutes). Although the distal colonic segment was continuously stimulated by the luminal distension, there was a resting period between successive PCs. Spontaneous PCs were produced every 90-100 seconds in the distal colon during the fluid distension. This suggests that underlying factors trigger peristaltic reflex. ${ }^{22}$

It is well established that the colonic peristaltic reflex is mediated by the release of 5-HT from enterochromaffin cells. ${ }^{32} \mathrm{~A}$ 5-HT acts $5-\mathrm{HT}_{4}$ and $5-\mathrm{HT}_{3}$ receptors to activate intramural sensory neurons that release calcitonin gene-related peptide. ${ }^{42}$ The presence of fecal pellets triggers the release of 5-HT, which acts via both $5-\mathrm{HT}_{3}$ and $5-\mathrm{HT}_{4}$ receptors to regulate propulsive activity of the guinea pig colon. ${ }^{28}$ Others showed that hexamethonium abolished the peristaltic reflex, suggesting the involvement of nicotinic receptors in the initiation of peristaltic reflex of the guinea pig colon. $^{29}$

In 1970, Crema et $\mathrm{al}^{36}$ demonstrated that atropine partially inhibited peristaltic reflex in response to intraluminal balloon distension of the guinea pig distal colon. The authors explained that the inhibitory effect of atropine is due to preventing the descending relaxation, because the inhibitory neurons responsible for descending relaxation are regulated by muscarinic receptors. ${ }^{36}$ However, currently it is well accepted that descending relaxations are regulated via nicotinic receptors, rather than muscarinic receptors. $^{32,43,44}$

Mucosal and muscle stimulation initiates peristalsis via ascending contractions and descending relaxations. Ascending contractions are mainly mediated via $\mathrm{ACh}$ and substance $\mathrm{P}$, while descending relaxations are mainly mediated via nitric oxide (NO), vasoactive intestinal polypeptide, and pituitary adenylate cyclase-activating peptide. ${ }^{45-47}$ Especially, released ACh and NO from the myenteric plexus play a crucial role in mediating peristalsis.

We have recently shown that $5-\mathrm{HT}_{3}$ and $5-\mathrm{HT}_{4}$ receptors promote colonic peristalsis via different mechanisms in guinea pigs. ${ }^{48}$ In the current study, we focused the possible role of muscarinic receptors, nicotinic receptors, and NO in mediating $\mathrm{PN}$-stimulated augmentation of peristaltic reflex of the guinea pig colon.

We have recently shown that L-NAME itself decreased the incidence of PCs to $26 \%$ and increased the incidence of NPCs to
$70 \%$ in response to luminal distension of the guinea pig distal colon in vitro. ${ }^{22}$ Similarly, our currently study showed that in the presence of L-NAME the incidence of PCs was reduced while the incidence of NPCs was increased in the distal colon. When PN stimulation was applied, non-coordinated NPCs were reversed to PCs even in the presence of L-NAME. This suggests that PN may recover the disorganized colonic activity due to blockade of $\mathrm{NO}$ neurons in the myenteric plexus, resulting in normal peristalsis.

ACh receptors are heavily implicated in PN stimulation effect due to complete inhibition during nicotinic or muscarinic blockade. Because $48 \%$ of pelvic ganglia neurons are NOS positive and most of these positive neurons co-express choline acetyltransferase (ChAT) in guinea pig, ${ }^{13}$ NOS blockade will predictably affect colonic motility significantly. It is likely that NOS co-expression on ChAT positive neurons serves to fine-tune the intensity of the excitatory stimulus, and inhibition of NOS signaling does not allow the organized, propagated peristalsis waves to develop due to overwhelming excitatory stimulus, which results in both coordinated and non-coordinated contractions.

In the past literature on peristalsis over a period of 40 years it has been clearly shown that in isolated guinea pig distal colon, nicotinic receptors are involved in augmentation of colonic peristalsis as hexamethonium abolished fecal pellet propulsion. ${ }^{29,36,49}$ In contrast, others demonstrated that nicotinic receptors are not essential in mediating peristalsis of the guinea pig distal colon. ${ }^{50,51}$ They showed that hexamethonium temporarily arrested peristalsis and fecal pellet propulsion. However, if time was allowed for the pellet to remain in the oral end of the colon, the pellet gradually propelled anally, until finally being expelled. During the initiation of peristalsis, hexamethonium significantly reduced the peak tension applied to the fecal pellet. ${ }^{50}$ The same group recently reported that propagation velocities are significantly reduced, but not abolished, in the presence of hexamethonium. ${ }^{51}$ These suggest that nicotinic receptors are partially involved in the regulation of peristalsis of the guinea pig distal colon. It seems that the contribution of nicotinic receptors to colonic peristalsis might depend upon experimental procedures. Our study demonstrated that hexamethonium and atropine completely inhibited intraluminal pressure increase in response to luminal infusion. $\mathrm{PN}$ stimulation failed to cause any PCs or NPCs in the presence of hexamethonium and atropine. This suggests that both muscarinic and nicotinic receptors are involved in augmentation of colonic peristalsis in response to $\mathrm{PN}$ stimulation.

In the current study, IMG stimulation resulted in a failure to 
increase intraluminal pressure and failure of pellet to progress along colonic segment suggesting the inhibitory mechanism of IMG stimulation on colonic peristalsis. It has been previously reported that sympathetic extrinsic stimulation in guinea pig colon caused an inhibitory effect on the normal colonic contractility. ${ }^{52} \mathrm{It}$ was also shown that sympathetic stimulation reduced the amount of ACh released during transmural or PN stimulation. ${ }^{52}$ Similarly, sympathetic nerve stimulation inhibits distal colorectal motility in several other animal models, including rabbits, ${ }^{53} \mathrm{dogs}^{34}$ and rats. $^{30}$

It has been shown that alpha- 2 receptor blockade accelerates colon transit and distal colonic motility ${ }^{54}$ and alpha- 2 receptor agonists decrease colonic activity in rats. ${ }^{55,56}$ The inhibitory pre-junctional alpha-2 receptors have been shown in the guinea pig ileum, ${ }^{57}$ dog small intestine ${ }^{34}$ and guinea pig stomach. ${ }^{58}$ The inhibitory effect of alpha-2 adrenoceptors on GI motility is through their inhibitory effect on pre-synaptic and post-ganglionic neurons, which are primarily cholinergic. Clinically, alpha-2 adrenergic agonist (clonidine) has been used in humans and shown to reduce colonic tone and improve symptoms in diarrhea-predominant IBS. ${ }^{59}$ In our experiment, the inhibitory effect of IMG stimulation on colonic peristalsis is partially restored by alpha- 2 receptor antagonist (yohimbine).

\section{Conclusions}

In summary, we have demonstrated that in guinea pig distal colon extrinsic autonomic nerves determine the speed of pellet propagation in vitro, though active pellet transit will occur without any extrinsic nerve input at baseline. This response depends on the activation of cholinergic and nitrergic neurons. In addition, stimulation of IMG significantly slowed down pellet transit to a fraction of propagation speed mediated by intrinsic reflexes. The inhibition of IMG stimulation is mediated by alpha-2 adrenoceptors.

This model has proven to yield predictable and highly consistent results, is cost effective, and easy to manipulate. In addition, the complexity of guinea pig pelvic innervation is higher than that of the rat and its active intrinsic system allows clear comparison of autonomic nerve stimulation effect. This model is highly useful for the study of autonomic nerve related colonic dysmotility, as well as effects of sacral nerve stimulation.

\section{Supplementary Material}

Note: To access the supplementary videos mentioned in this article, visit the online version of Journal of Neurogastroenterology and Motility at http://www.jnmjournal.org/, and at doi: http://dx.doi.org/10.5056/jnm.2014.20.2.185.

\section{References}

1. Jackson KS, Naik R. Pelvic floor dysfunction and radical hysterectomy. Int J Gynecol Cancer 2006;16:354-363.

2. Vierhout ME, Schreuder HW, Veen HF. Severe slow-transit constipation following radical hysterectomy. Gynecol Oncol 1993;51: 401-403.

3. Bryant CL, Lunniss PJ, Knowles CH, Thaha MA, Chan CL. Anterior resection syndrome. Lancet Oncol 2012;13:e403-e408.

4. Speakman CT, Kamm MA. Abnormal visceral autonomic innervation in neurogenic faecal incontinence. Gut 1993;34:215-221.

5. Leroi AM, Damon H, Faucheron JL, et al. Sacral nerve stimulation in faecal incontinence: position statement based on a collective experience. Colorectal Dis 2009;11:572-583.

6. Thomas GP, Dudding TC, Rahbour G, Nicholls RJ, Vaizey CJ Sacral nerve stimulation for constipation. Br J Surg 2013;100:174181.

7. Shimizu K, Koda K, Kase Y, et al. Induction and recovery of colonic motility/defecatory disorders after extrinsic denervation of the colon and rectum in rats. Surgery 2006;139:395-406.

8. Lee WY, Takahashi T, Pappas T, Mantyh CR, Ludwig KA. Surgical autonomic denervation results in altered colonic motility: an explanation for low anterior resection syndrome? Surgery 2008;143: 778-783.

9. Maruyama S, Okabe S, Endo M, Sato K, Iwai T. The role of the rectal branches of pelvic plexus in defecation and colonic motility in a canine model. J Med Dent Sci 2003;50:275-284.

10. Ridolfi T, Tong W, Kosinski L, Takahashi T, Ludwig K. Recovery of colonic transit following extrinsic nerve damage in rats. Scand J Gastroenterol 2011;46:678-683.

11. Gribovskaja-Rupp I, Takahashi T, Ridolfi T, Kosinski L, Ludwig $\mathrm{K}$. Upregulation of mucosal $5-\mathrm{HT}_{3}$ receptors is involved in restoration of colonic transit after pelvic nerve transection. Neurogastroenterol Motil 2012;24:472-478, e218.

12. Tong W, Kamiyama Y, Ridolfi TJ, et al. The role of $5-\mathrm{HT}_{3}$ and 5-HT $\mathrm{H}_{4}$ receptors in the adaptive mechanism of colonic transit following the parasympathetic denervation in rats. J Surg Res 2011;171: 510-516.

13. Olsson C, Chen BN, Jones S, Chataway TK, Costa M, Brookes SJ. Comparison of extrinsic efferent innervation of guinea pig distal colon and rectum. J Comp Neurol 2006;496:787-801.

14. Dhami D, Mitchell BS. Specific patterns of immunoreactivity in neuronal elements of the anterior major pelvic ganglion of the male guinea-pig. J Anat 1991;176:197-210.

15. Mitchell BS. Morphology and neurochemistry of the pelvic, and paracervical ganglia. Histol Histopathol 1993;8:761-773. 
16. Yokota R, Burnstock G. Decentralisation of neurones in the pelvic ganglion of the guinea-pig: reinnervation by adrenergic nerves. Cell Tissue Res 1983;232:399-411.

17. Mazzanti L, Del Tacca M, Breschi MC, Frigo GM, Friedman C, Crema A. The time course of functional and morphological changes of the guinea-pig colon after "a frigore" denervation of the periarterial sympathetic nerves. Acta Neuropathol 1972;22:190-199.

18. Marino F, Marcoli M, Lecchini S, Frigo GM. Supersensitivity to morphine after chronic sympathetic denervation in guinea-pig colon. J Pharm Pharmacol 1992;44:526-527.

19. Giaroni C, Zanetti E, Pascale A, et al. Involvement of $\mathrm{Ca}^{2+}$-dependent PKCs in the adaptive changes of mu-opioid pathways to sympathetic denervation in the guinea pig colon. Biochem Pharmacol 2009; 78:1233-1241.

20. Yokota R, Burnstock G. Synaptic organisation of the pelvic ganglion in the guinea-pig. Cell Tissue Res 1983;232:379-397.

21. Yoneda S, Kadowaki M, Kuramoto H, Fukui H, Takaki M. Enhanced colonic peristalsis by impairment of nitrergic enteric neurons in spontaneously diabetic rats. Auton Neurosci 2001;92:65-71.

22. Kwak JM, Babygirija R, Gribovskaja-Rupp I, Takahashi T, Yamato $\mathrm{S}$, Ludwig K. Regional difference in colonic motility response to electrical field stimulation in Guinea pig. J Neurogastroenterol Motil 2013;19:192-203.

23. Takahashi T, Owyang C. Regional differences in the nitrergic innervation between the proximal and the distal colon in rats. Gastroenterology 1998;115:1504-1512.

24. Alberti E, Mikkelsen HB, Larsen JO, Jimenez M. Motility patterns and distribution of interstitial cells of Cajal and nitrergic neurons in the proximal, mid- and distal-colon of the rat. Neurogastroenterol Motil 2005;17:133-147.

25. Hasler WL, Kurosawa S, Chung OY. Regional cholinergic differences between distal and proximal colonic myenteric plexus. Am J Physiol 1990;258:G404-G410.

26. D'Antona G, Hennig GW, Costa M, Humphreys CM, Brookes SJ. Analysis of motor patterns in the isolated guinea-pig large intestine by spatio-temporal maps. Neurogastroenterol Motil 2001;13:483-492.

27. Foxx-Orenstein AE, Jin JG, Grider JR. 5-HT $\mathrm{H}_{4}$ receptor agonists and delta-opioid receptor antagonists act synergistically to stimulate colonic propulsion. Am J Physiol 1998;275:G979-G983.

28. Jin JG, Foxx-Orenstein AE, Grider JR. Propulsion in guinea pig colon induced by 5 -hydroxytryptamine (HT) via $5-\mathrm{HT}_{4}$ and $5-\mathrm{HT}_{3}$ receptors. J Pharmacol Exp Ther 1999;288:93-97.

29. Kadowaki M, Wade PR, Gershon MD. Participation of $5-\mathrm{HT}_{3}$, $5-\mathrm{HT}_{4}$, and nicotinic receptors in the peristaltic reflex of guinea pig distal colon. Am J Physiol 1996;271:G849-G857.

30. Ridolfi TJ, Tong WD, Takahashi T, Kosinski L, Ludwig KA. Sympathetic and parasympathetic regulation of rectal motility in rats. J Gastrointest Surg 2009;13:2027-2033.

31. Tong WD, Ridolfi TJ, Kosinski L, Ludwig K, Takahashi T. Effects of autonomic nerve stimulation on colorectal motility in rats. Neurogastroenterol Motil 2010;22:688-693.

32. Grider JR, Jin JG. Distinct populations of sensory neurons mediate the peristaltic reflex elicited by muscle stretch and mucosal stimulation. J Neurosci 1994;14:2854-2860.

33. Spencer NJ, Smith TK. Mechanosensory S-neurons rather than AH-neurons appear to generate a rhythmic motor pattern in guin- ea-pig distal colon. J Physiol 2004;558:577-596.

34. Hughes SF, Scott SM, Pilot MA, Williams NS. Adrenoceptors and colocolonic inhibitory reflex. Dig Dis Sci 1999;44:2462-2468.

35. Ishikawa M, Mibu R, Iwamoto T, Konomi H, Oohata Y, Tanaka $\mathrm{M}$. Change in colonic motility after extrinsic autonomic denervation in dogs. Dig Dis Sci 1997;42:1950-1956.

36. Crema A, Frigo GM, Lecchini S. A pharmacological analysis of the peristaltic reflex in the isolated colon of the guinea-pig or cat. $\mathrm{Br} \mathrm{J}$ Pharmacol 1970;39:334-345.

37. Frigo GM, Lecchini S. An improved method for studying the peristaltic reflex in the isolated colon. Br J Pharmacol 1970;39:346-356.

38. DeGroat WC, Saum WR. Synaptic transmission in parasympathetic ganglia in the urinary bladder of the cat. J Physiol 1976;256:137-158.

39. Rao GN, Drew PJ, Lee PW, Monson JR, Duthie GS. Anterior resection syndrome is secondary to sympathetic denervation. Int $\mathrm{J}$ Colorectal Dis 1996;11:250-258.

40. Krier J. Motor function of anorectum and pelvic floor musculature. Compr Physiol 2011:1025-1053. Available URL from: http:// onlinelibrary.wiley.com/doi/10.1002/cphy.cp060127/full (accessed 24 Feb 2014).

41. Hirabayashi T, Matsufuji H, Yokoyama J, et al. Colorectal motility induction by sacral nerve electrostimulation in a canine model: implications for colonic pacing. Dis Colon Rectum 2003;46:809-817.

42. Grider JR. CGRP as a transmitter in the sensory pathway mediating peristaltic reflex. Am J Physiol 1994;266:G1139-G1145.

43. Smith TK, McCarron SL. Nitric oxide modulates cholinergic reflex pathways to the longitudinal and circular muscle in the isolated guinea-pig distal colon. J Physiol 1998;512 (Pt 3):893-906.

44. Gribovskaja-Rupp I, Kwak JM, Takahashi T, Ludwig K. Nitric oxide regulates polarity of guinea pig distal colon pellet propagation and circular muscle motor response. J Gastroenterol 2013 (In press)

45. Foxx-Orenstein AE, Grider JR. Regulation of colonic propulsion by enteric excitatory and inhibitory neurotransmitters. Am J Physiol 1996;271:G433-G437.

46. Grider JR. Interplay of VIP and nitric oxide in regulation of the descending relaxation phase of peristalsis. Am J Physiol 1993;264: G334-G340.

47. Murthy KS, Jin JG, Grider JR, Makhlouf GM. Characterization of PACAP receptors and signaling pathways in rabbit gastric muscle cells. Am J Physiol 1997;272:G1391-G1399.

48. Gribovskaja-Rupp I, Kwak J, Takahashi T, Ludwig K. 5-HT 3 and 5-HT $\mathrm{H}_{4}$ receptors promote colonic peristalsis via different mechanisms in guinea pigs. DDW 2012 May 19-22, San Diego Convention Center, San Diego, CA: A465.

49. Tonini M, Spelta V, De Ponti F, et al. Tachykinin-dependent and -independent components of peristalsis in the guinea pig isolated distal colon. Gastroenterology 2001;120:938-945.

50. Nicholas S, Spencer NJ. Peristalsis and fecal pellet propulsion do not require nicotinic, purinergic, $5-\mathrm{HT}_{3}$, or $\mathrm{NK}_{3}$ receptors in isolated guinea pig distal colon. Am J Physiol Gastrointest Liver Physiol 2010;298:G952-G961.

51. Sia TC, Brookes SJ, Dinning PG, Wattchow DA, Spencer NJ. Peristalsis and propulsion of colonic content can occur after blockade of major neuro-neuronal and neuro-muscular transmitters in isolated guinea-pig colon. Am J Physiol Gastrointest Liver Physiol 2013;305: G933-G939. 
52. Beani L, Bianchi C, Crema A. The effect of catecholamines and sympathetic stimulation on the release of acetylcholine from the guinea-pig colon. Br J Pharmacol 1969;36:1-17.

53. Gillespie JS, Khoyi MA. The site and receptors responsible for the inhibition by sympathetic nerves of intestinal smooth muscle and its parasympathetic motor nerves. J Physiol 1977;267:767-789.

54. Theodorou V, Fioramonti, Bueno L. Central $\alpha_{2}$-adrenergic control of colonic transit in rats.Neurogastroenterol Motil Published Online First: 28 Jun 2008. doi: 10.1111/j.1365-2982.1989.tb00145.x

55. Sjöqvist A, Hallerback B, Glise $H$. Reflex adrenergic inhibition of colonic motility in anesthetized rat caused by nociceptive stimuli of peritoneum. An alpha 2-adrenoceptor-mediated response. Dig Dis Sci 1985;30:749-754.
56. Salaymeh BM, Cowles VE, Tekin E, Zhu YR, Browne BJ, Condon RE. Selective adrenergic agonists and colon motility in monkeys. Surgery 1992;111:694-698.

57. Bauer V. Distribution and types of adrenoceptors in the guinea-pig ileum: the action of alpha- and beta-adrenoceptor blocking agents. $\mathrm{Br}$ J Pharmacol 1982;76:569-578.

58. Sahyoun HA, Costall B, Naylor RJ. Catecholamines act as alpha 2-adrenoceptors to cause contraction of circular smooth muscle of guinea-pig stomach. J Pharm Pharmacol 1982;34:381-385.

59. Bharucha AE, Seide BM, Zinsmeister AR. The effects of clonidine on symptoms and anorectal sensorimotor function in women with faecal incontinence. Aliment Pharmacol Ther 2010;32:681-688. 